\section{New gene expression map of kidney development}

A new atlas of gene expression during multiple stages of nephron formation in the developing mouse kidney has been mapped out, representing the first genomics-level analysis of organogenesis in a mammalian organ. Comparison of this atlas with gene expression patterns in mutant mice might help the understanding of abnormalities in kidney development.

Laser capture microdissection or fluorescence-activated cell sorting were used to isolate and purify discrete cell populations in kidneys from 15.5-day-old embryonic transgenic mice. Given that new nephrons form on the surface of the developing kidney and mature nephrons are located deep within the structure, samples taken from a single time point could be used to examine gene expression states in multiple stages of nephron formation.

Microarray profiling was used to identify probe sets specific for 15 embryonic compartments of the developing kidney, which represented multiple developmental stages of the nephron. Assessment of the differential expression of genes identified 7,629 probe sets, with adjacent compartments in the developing kidney showing high levels of correlative gene expression. Few compartments, however, exhibited highly restricted gene expression patterns, bar the proximal tubule and the ureteral smooth muscle. The quality of the microarray data was then confirmed by in silico comparison with previously determined gene expression patterns and by in situ hybridization.

These results indicate that kidney development follows an analog model in which levels of gene expression are important, rather than a digital model based on an on-off pattern of gene expression.

Original article Brunskill EW et al. (2008) Atlas of gene expression in the developing kidney at microanatomic resolution. Dev Cell 15: 781-791

\section{MRL can accurately detect nodal metastases in patients with prostate cancer}

Diagnostic pelvic lymph-node dissection (PLND) is the gold standard for detection of nodal metastases in patients with prostate cancer. Introduction of the lymph-node-specific intravenous contrast agent, ferumoxtran-10, has increased the potential accuracy of CT and MRI for detecting metastases. Heesakkers and colleagues carried out a prospective, multicohort study to compare the diagnostic accuracy of multidetector CT (MDCT) with that of magnetic resonance lymphangiography (MRL), and to investigate whether a negative MRL result can preclude the need for PLND.

A total of 375 patients with prostate cancer who had an intermediate or high risk of lymphnode metastases were enrolled in the study. In relation to the detection of lymph-node metastases, the sensitivity of MDCT and MRL were $34 \%$ and $82 \%$, respectively. Specificity was $97 \%$ for MDCT compared with $93 \%$ for MRL. The negative predictive values for MDCT and MRL were $88 \%$ and $96 \%$, respectively, and positive predictive values were $66 \%$ and $69 \%$, respectively. A total of 61 patients had lymphnode metastases, and in 50 patients these metastases were detected by MRL. In at least $30 \%$ of patients with lymph-node metastases, positive nodes were detected only by MRL, as they were outside the routine PLND area.

The authors conclude that MRL has higher sensitivity than MDCT to detect potential lymph-node metastases in patients with prostate cancer. In patients with negative MRL findings, the probability of lymph-node metastases is low enough to preclude PLND.

Original article Heesakkers RA et al. (2008) MRI with a lymph-node-specific contrast agent as an alternative to CT scan and lymph-node dissection in patients with prostate cancer: a prospective multicohort study. Lancet Oncol 9: $850-856$ 